\title{
Active surveillance scheme in three Romanian hospitals reveals a high prevalence and variety of carbapenamase- producing Gram-negative bacteria: a pilot study, December 2014 to May 2015
}

D Timofte 1234 , CV Panzaru 457 , IE Maciuca ${ }^{14}$, M Dan 7 , AD Mare 6 , A Man 6 , F Toma 6

1. School of Veterinary Science, University of Liverpool, Leahurst Campus, Neston, United Kingdom

2. Institute of Infection and Global Health, University of Liverpool, Liverpool, United Kingdom

3. Faculty of Veterinary Medicine, University of Agronomical Sciences and Veterinary Medicine, Iasi, Romania

4. These authors contributed equally to this work

5. Microbiology Department, University of Medicine and Pharmacy 'Grigore T. Popa', Iasi, Romania

6. Microbiology Department, University of Medicine and Pharmacy Targu Mures, Targu Mureș, Romania

7. Institute of Cardiovascular Diseases, Iasi, Romania

Correspondence: Dorina Timofte (d.timofte@liverpool.ac.uk)

Citation style for this article:

Timofte D, Panzaru CV, Maciuca IE, Dan M, Mare AD, Man A, Toma F. Active surveillance scheme in three Romanian hospitals reveals a high prevalence and variety of carbapenamase-producing Gram-negative bacteria: a pilot study, December 2014 to May 2015. Euro Surveill. 2016;21(25):pii=30262. DOI: http://dx.doi.

org/10.2807/1560-7917.ES.2016.21.25.30262

Article submitted on 30 September 2015 / accepted on 12 February 2016 / published on 23 June 2016

We report the findings of an active surveillance scheme for detection of asymptomatic carriers with carbapenemase-producing Gram-negative bacteria (CP-GNB) in Romanian hospitals. During a pilot study from December 2014 to May 2015, faecal cultures were screened in three hospitals (two large, one mediumsize) for patients newly admitted to selected wards or inpatients transferred from other wards to an intensive-care unit. The study revealed a high prevalence of CP-GNB detected in $22 / 27$ and $28 / 38$ of the carbapenem non-susceptible isolates from Hospitals 1 and 3 , respectively. CP-GNB identified through faecal screening included NDM-1-producing Serratia marcescens and Klebsiella pneumoniae, OXA-48-producing $K$. pneumoniae and OXA-23-producing Acinetobacter baumannii. The distribution of the CP-GNB varied between the hospitals, with NDM-1-producing $S$. marcescens and $K$. pneumoniae being prevalent in the northcentral part of the country and OXA-23/24-producing A. baumannii, OXA-48-producing K.pneumoniae, Morganella morganii and VIM-2-producing Escherichia coli/Pseudomonas aeruginosa detected in the northeast of the country. Conjugation studies showed that carbapenem resistance was transferable and PCRbased replicon typing identified blaNDM-1 on IncFIls in S. marcescens and $K$. pneumoniae from Hospital 1 and blaOXA-48 on IncL plasmids in all Klebsiella spp. isolates from Hospitals 1 and 3. Our findings underline the importance of active surveillance for detection of CP-GNB asymptomatic faecal carriers and suggest a likely endemic spread of CP-GNB in Romania.

\section{Introduction}

The worldwide emergence of carbapenemase-producing Gram-negative bacteria (CP-GNB) is widely accepted as a major public health threat that has caused international concern. The rapid spread of CP-GNB provides many challenges for healthcare systems, with implications that span from laboratory detection and infection control, to identifying suitable treatment options for organisms that are now starting to exhibit co-resistance to multiple last-resort antimicrobials [1].

The epidemiology of CP-GNB in Europe varies between northern and southern countries. A European survey of carbapenemase-producing Enterobacteriaceae (EUSCAPE) among national experts from 39 European countries in 2013 showed that carbapenemase-producing Enterobacteriaceae (CPE) were continuing to spread in Europe, with Klebsiella pneumoniae carbapenemase (KPC)-producing isolates having the widest distribution and the number of OXA-48-producing isolates continuing to rise [2]. In contrast, NDM-1-producing isolates are sporadically reported, mainly from the United Kingdom (UK) [2]. The same report also found that 30 of the 39 European countries had a dedicated surveillance system for CPE and 22 of the countries had national recommendations or specific guidelines for infection control [2]; however, Romania does not have such a surveillance system or CPE infection control guidelines.

Romania contributes to the European Antimicrobial Resistance Surveillance Network (EARS-Net) although only via a small number of laboratories that report only carbapenem-resistant invasive $K$. pneumoniae and 


\section{FIGURE 1}

Genes associated with carbapenem resistance (here including bla ${ }_{\text {id }}$ ) identified in two hospitals, northcentral (Hospital 1, 22 isolates) and north-east Romania (Hospital 3, 28 isolates), December 2014-May 2015

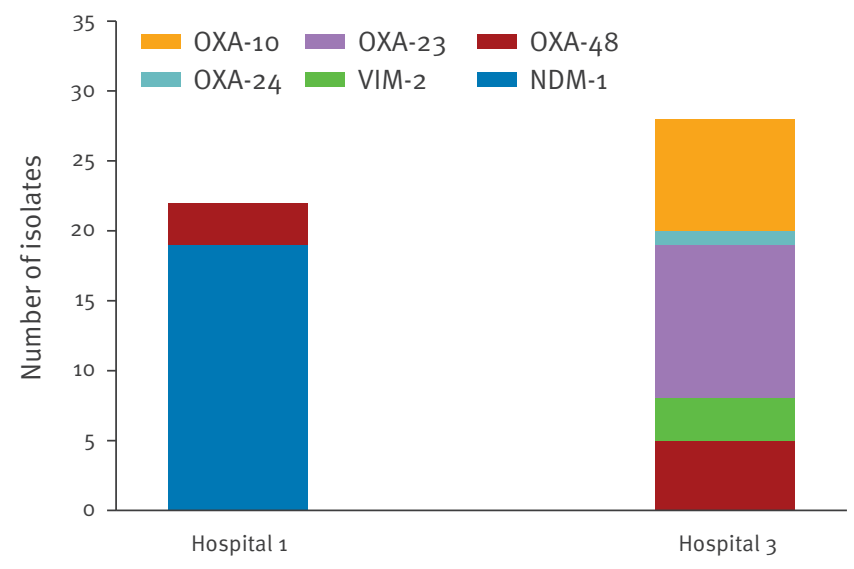

Escherichia coli from blood and cerebrospinal fluid. As a result, most European epidemiological reports show that data on CP-GNB from Romania are unknown or uncertain [3]. To get a snap-shot of the epidemiology of CP-GNB in hospitals that do not report to EARS-Net and that were not included in the 2013 EuSCAPE, a pilot CP-GNB surveillance project - introducing faecal screening for carbapenemase-producing bacteria on admission of patients to hospital and following transfer of inpatients from other wards to an intensive-care unit (ICU), as well as screening of clinical isolates was implemented in three Romanian hospitals during December 2014 to May 2015. Few studies have investigated intestinal colonisation with carbapenemaseproducing bacteria at the point of hospital admission: here we report the findings of an active surveillance programme for carbapenemase-producing bacteria in three hospitals in Romania.

\section{Methods}

Microbiology laboratories from three Romanian hospitals participated in the study. The hospitals were from two different geographical regions, with two hospitals (Hospitals 1 and 2) from the north-central part of the country, while the third (Hospital 3) was from the north-east of Romania. Hospitals 1 and 2 were large, with 1,299 and 1,892 beds, respectively, while Hospital 3 had a smaller capacity, with 232 beds and about 1,900 patients treated each year (the annual number of patients treated in Hospitals 1 and 2 could not obtained). Ethical approval for the study was obtained from the Hospital Research Ethics Committee at each hospital. Written consent was obtained from all patients for biological sample collection for routine investigation according to the hospitals' protocols.
During the study period (December 2014 to May 2015), surveillance of faecal cultures was introduced in the routine admission procedures as part of infection prevention and control measures. Each hospital analysed faecal cultures within the first 48 hours of hospitalisation for patients newly admitted to selected wards (such as haematology units with transplant recipients, ICUs or cardiac ICUs, nephrology and neonatology, gastroenterology, infectious diseases units and several surgical specialities) and also for inpatients transferred from other wards to an ICU. Screening criteria for newly admitted patients, if applied by the hospital, included previous hospitalisation, previous infections with multidrug-resistant bacteria (if known) or previous antimicrobial treatment.

Carbapenem non-susceptible clinical isolates (from blood, urine, tracheal secretions) obtained from patients from the selected wards, were also included in the surveillance and when detected, these patients and their contacts were also checked for faecal colonisation. Suspicion of an ongoing outbreak with a CP-GNB during the study period in Hospital 1 lead to environmental samples being collected, which were also included in the study.

Carbapenemase screening protocols for Enterobacteriaceae and non-fermenting Gram-negative organisms were considered and a single protocol was used in the participating hospitals. For screening of faecal samples, a small sample inoculum was placed in $5 \mathrm{~mL}$ trypticase soy broth (TSB) containing a meropenem $(10 \mu \mathrm{g})$ disc and incubated at $37^{\circ} \mathrm{C}$ overnight. The following day, $100 \mu \mathrm{L}$ of TSB was transferred onto a MacConkey agar plate (all discs and media were from Oxoid, Basingstoke, UK) and one disc each of ertapenem $(10 \mu \mathrm{g})$ and meropenem (10 $\mu \mathrm{g})$ were placed on different sectors of the inoculum. Clinical breakpoints and screening cut-off values for CPE were according to the European Committee on Antimicrobial Susceptibility (EUCAST) methodology [4]. All carbapenem non-susceptible isolates were identified (VITEK 2 system, Biomerieux, France) and stored at $-80{ }^{\circ} \mathrm{C}$ for further testing.

Molecular characterisation of isolates was performed at Liverpool University, UK, which consisted of screening for resistance markers by multiplex PCR followed by sequencing. The PCR included screening for extended spectrum beta-lactamases (ESBLs) including bla $a_{\text {CTX- }}$ M, bla $a_{\text {SHV }}, b l a_{\text {TEM, }}, a_{\text {OXA }}$ and carbapenem-resistance genes

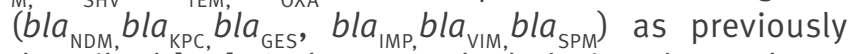
described [5-7]. Carbapenem-hydrolysing class $D$ betalactamases (CHDLs) corresponding to OXA-23, OXA24/40, OXA-48, OXA-51, OXA-58 and OXA-148 groups, as well as OXA-10, previously described as narrowspectrum and recently shown to have CHDL activity in Acinetobacter spp. $[8,9]$, were also included. Positive and negative controls were included in all PCR reactions. Minimum inhibitory concentration (MIC) testing for isolates with a unique genotype was performed by 
Carbapenemase-producing Gram-negative bacterial species $(n=5)$ and distribution of carbapenemase- and beta-lactamase genes $(n=8)$ identified in each bacterial species in Hospital 3 (28 isolates), north-east Romania, December 2014-May 2015

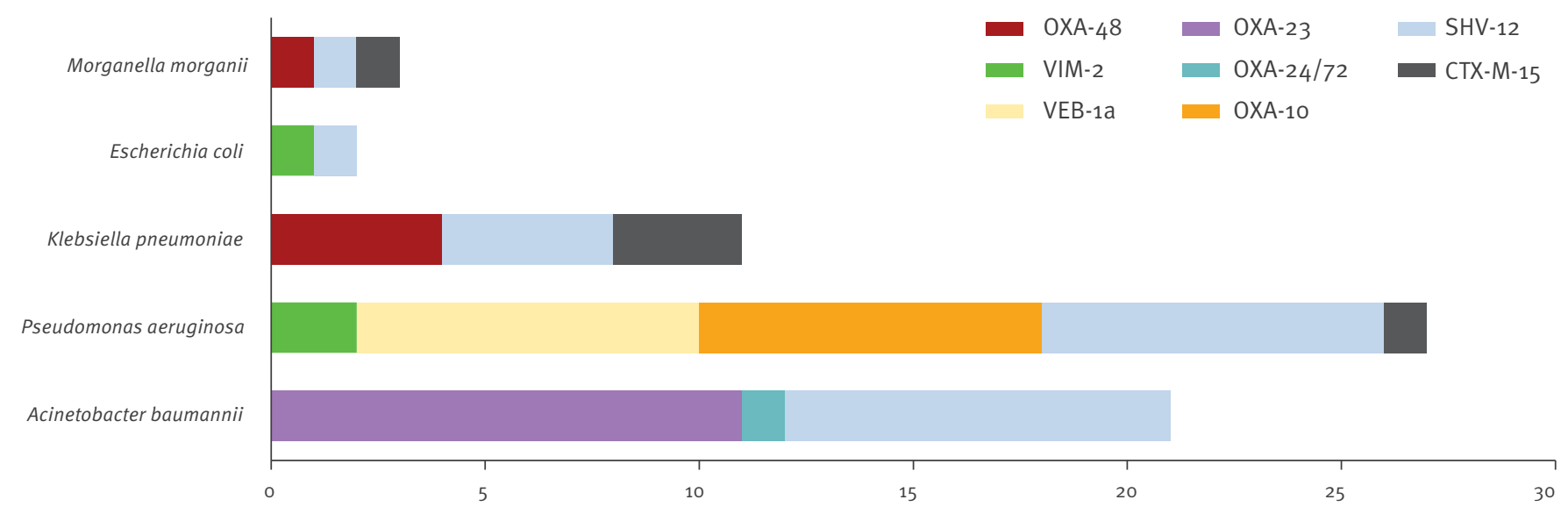

Distribution of carbapenemase/beta-lactamase genes in each bacterial species

broth microdilution with Sensititre GNX3F plates (TREK Diagnostic Systems, West Sussex, UK). The results were interpreted according to EUCAST breakpoints for all antimicrobials except for polymyxin B, where Clinical and Laboratory Standard Institute (CLSI) criteria were applied $[10,11]$.

To determine whether the carbapenem-resistance genes were transferable, conjugation was performed by broth mating as previously described [12] and was attempted for all CPE identified in this study. Donor and recipient cells (plasmid-free streptomycin-resistant $E$. coli $\mathrm{HB} 101)$ were grown to logarithmic phase, mixed in nutrient broth and incubated at $37^{\circ} \mathrm{C}$ for 18 hours. Transconjugants were selected on nutrient agar (Oxoid, UK) supplemented with streptomycin $(50 \mu \mathrm{g} / \mathrm{mL})$ and cefotaxime $(1 \mu \mathrm{g} / \mathrm{mL})$. To ensure that selected isolates were the recipient $E$. coli and that they contained the transferred resistance gene, PCR was performed for the uidA gene [13] as well as carbapenemase-resistance genes. In addition, PCR was performed to identify if $\mathrm{ESBL}$ resistance determinants were co-transferred with the carbapenem-resistance. Plasmid DNA was extracted from clinical isolates and transconjugants using a QIAprep spin miniprep kit (QIAGEN, Germany). Plasmid identification using a PCR-based replicon typing (PBRT) scheme [14] was carried out on total DNA from all CPE clinical isolates carrying carbapenemresistance genes, as well as on plasmid DNA extracted from the clinical isolates and their transconjugants. It was previously observed that PBRT can be inefficient at identifying IncL/M plasmid type, therefore an up-dated method designed to identify and distinguish between IncL and IncM plasmids was used $[14,15]$.

\section{Results}

A total of 820 clinical, faecal and environmental specimens were analysed: 528 samples were from Hospital 1 (7 clinical, 336 faecal, 185 environmental), 105 from
Hospital 2 (all faecal) and 187 from Hospital 3 (23 clinical, 164 faecal). Carbapenem non-susceptible Gram-negative isolates were identified in $27 / 528$ (5\%), $12 / 102(12 \%)$ and $38 / 187(20 \%)$ of the samples from Hospitals 1, 2 and 3, respectively. Molecular testing identified carriage of at least one gene associated with carbapenem resistance in $22 / 27$ of the carbapenem non-susceptible isolates from Hospital 1, none from Hospital 2 and 28/38 isolates from Hospital 3 (Figure 1).

A total of 19 isolates from Hospital 1 carried bla ${ }_{\mathrm{NDM}-1}$ (17 Serratia marcescens and two K. pneumoniae) while three isolates (all K. pneumoniae) carried bla $a_{0 \times A-48}$. The NDM-1 positive isolates were from clinical specimens (seven isolates from pharyngeal secretions), faecal screening (nine isolates) and hospital environmental samples (three isolates, from feeding tube, bedside and food jar) while the OXA-48-producing $K$. pneumoniae isolates were all from faecal screening. Of the 19 NDM-1-positive isolates, 15 co-harboured bla ${ }_{\text {CTX-M-15 }}$ while two produced LEN-25 beta-lactamases. Moreover, an unexpected variety of species carrying one or sometimes two genes associated with carbapenem resistance were identified in Hospital 3 (Figure 2).

A total of 11 A. baumannii isolates from Hospital 3 (nine from clinical samples (blood, tracheal secretions, urine) and two faecal isolates) were found to harbour bla $a_{\mathrm{OXA}-23}$ while another clinical isolate carried bla $a_{\mathrm{OXA}-24 / 27^{\circ}}$ The second most prevalent gene associated with carbapenem resistance in this hospital was bla ${ }_{\text {OXA-48 }}$, detected in clinical isolates of $K$. pneumoniae $(\mathrm{n}=4)$ and Morganella morganii $(\mathrm{n}=1)$. In addition, two Pseudomonas aeruginosa clinical isolates carried bla $a_{\text {VIM-2 }}$ while eight $P$. aeruginosa isolates (six faecal, two clinical) co-harboured bla $a_{\mathrm{OXA}-10}$ and bla $a_{\mathrm{VEB}-1 \mathrm{a}}$ betalactamase. Futhermore, one patient was found to have faecal colonisation with $E$. coli-producing VIM-2. KPC 
Antibiotic susceptibility testing of selected Klebsiella pneumoniae, Serratia marcescens, Acinetobacter baumannii and Pseudomonas aeruginosa isolates, from two hospitals, north-central and north-east Romania, December $2014-$ May 2015 (n $=18)$

\begin{tabular}{|c|c|c|c|c|c|c|c|c|c|c|c|c|c|c|c|c|c|c|c|c|c|c|}
\hline $\begin{array}{l}\text { Isolate ID } \\
\text { (Source) }\end{array}$ & Species & Genotype & AK & SAM & ATM & FEP & CTX & $C A Z$ & $\mathrm{CIP}$ & CST & DOR & DOX & GEN & IMP & LVX & MEM & TZP & PMB & TIM & TGC & TOB & SXT \\
\hline 8TM (F) & $\begin{array}{c}\text { K. } \\
\text { pneumoniae }\end{array}$ & $\begin{array}{c}\text { OXA-48, } \\
\text { CTX-M-15, } \\
\text { SHV-11 }\end{array}$ & $S$ & $\mathrm{R}$ & $\mathrm{R}$ & $\mathrm{R}$ & $\mathrm{R}$ & $R$ & $\mathrm{R}$ & S & S & $\mathrm{R}$ & $\mathrm{R}$ & $S$ & $\mathrm{R}$ & $S$ & $S$ & $S$ & $\mathrm{R}$ & S & $R$ & S \\
\hline 6TM (F) & $\begin{array}{c}\text { K. } \\
\text { pneumoniae }\end{array}$ & $\begin{array}{l}\text { NDM-1, } \\
\text { LEN-25 }\end{array}$ & $R$ & $\mathrm{R}$ & 1 & $R$ & $R$ & $\mathrm{R}$ & $S$ & S & $R$ & $\mathrm{R}$ & $\mathrm{R}$ & $R$ & $S$ & $\mathrm{R}$ & $R$ & $S$ & $\mathrm{R}$ & S & $R$ & $S$ \\
\hline 5TM (F) & $\begin{array}{c}\text { K. } \\
\text { pneumoniae }\end{array}$ & $\begin{array}{l}\text { NDM-1, } \\
\text { CTX-M-15 }\end{array}$ & $R$ & $\mathrm{R}$ & $\mathrm{R}$ & $\mathrm{R}$ & $R$ & $R$ & $R$ & $R$ & $\mathrm{R}$ & $R$ & $R$ & $R$ & $S$ & $R$ & $R$ & $R$ & $R$ & S & $R$ & $\mathrm{R}$ \\
\hline 4TM (F) & $\begin{array}{c}\text { S. } \\
\text { marcescens }\end{array}$ & $\begin{array}{c}\text { NDM-1, } \\
\text { CTX-M-15 }\end{array}$ & $R$ & $\mathrm{R}$ & $\mathrm{R}$ & $R$ & $R$ & $\mathrm{R}$ & $\mathrm{R}$ & $R$ & $S$ & S & $\mathrm{R}$ & $S$ & $S$ & $\mathrm{~S}$ & $\mathrm{R}$ & $\mathrm{R}$ & $\mathrm{R}$ & $S$ & $\mathrm{R}$ & $\mathrm{R}$ \\
\hline $1 T M(F)$ & $\begin{array}{c}\text { S. } \\
\text { marcescens }\end{array}$ & $\begin{array}{l}\text { NDM-1, } \\
\text { LEN-25 }\end{array}$ & $\mathrm{R}$ & $R$ & S & NT & $\mathrm{R}$ & $R$ & S & S & $R$ & $\mathrm{R}$ & $R$ & $R$ & $S$ & $R$ & $R$ & $S$ & $R$ & S & $R$ & $S$ \\
\hline $44 \mathrm{I}(\mathrm{F})$ & A. baumannii & $\begin{array}{l}\text { OXA-23, } \\
\text { SHV-12 }\end{array}$ & $\mathrm{R}$ & $\mathrm{R}$ & NT & NT & NT & $\mathrm{R}$ & $R$ & S & $\mathrm{R}$ & $\mathrm{R}$ & $R$ & $R$ & $\mathrm{R}$ & $\mathrm{R}$ & $R$ & S & $R$ & S & $R$ & $S$ \\
\hline $431(\mathrm{CL})$ & $\begin{array}{c}\text { K. } \\
\text { pneumoniae }\end{array}$ & $\begin{array}{l}\text { OXA-48, } \\
\text { SHV-11 }\end{array}$ & S & $\mathrm{R}$ & $R$ & S & $R$ & $R$ & $R$ & S & S & $R$ & S & S & $R$ & S & $R$ & S & $R$ & S & $\mathrm{R}$ & $S$ \\
\hline $40 \mathrm{l}(\mathrm{CL})$ & A. baumannii & $\begin{array}{c}\text { OXA-23, } \\
\text { SHV-1/28, } \\
\text { CTX-M-15 }\end{array}$ & $R$ & $\mathrm{R}$ & NT & NT & NT & NT & $\mathrm{R}$ & S & $R$ & $\mathrm{R}$ & $\mathrm{R}$ & $\mathrm{R}$ & $\mathrm{R}$ & $\mathrm{R}$ & $\mathrm{R}$ & S & $\mathrm{R}$ & S & $\mathrm{R}$ & $S$ \\
\hline 73 l (CL) & $\begin{array}{c}\text { K. } \\
\text { pneumoniae }\end{array}$ & $\begin{array}{c}\text { OXA-48, } \\
\text { CTX-M-15, } \\
\text { SHV-1/28 } \\
\end{array}$ & $\mathrm{R}$ & $\mathrm{R}$ & $R$ & $\mathrm{R}$ & $\mathrm{R}$ & $\mathrm{R}$ & $\mathrm{R}$ & $R$ & $R$ & $\mathrm{R}$ & $\mathrm{R}$ & $\mathrm{R}$ & $R$ & $\mathrm{R}$ & $\mathrm{R}$ & $\mathrm{R}$ & $\mathrm{R}$ & $\mathrm{S}$ & $\mathrm{R}$ & $S$ \\
\hline 71 I (CL) & $\begin{array}{c}\text { K. } \\
\text { pneumoniae }\end{array}$ & $\begin{array}{c}\text { OXA-48, } \\
\text { CTX-M-15, } \\
\text { SHV-1/28 }\end{array}$ & $\mathrm{R}$ & $\mathrm{R}$ & $\mathrm{R}$ & $R$ & $\mathrm{R}$ & $\mathrm{R}$ & $\mathrm{R}$ & $\mathrm{R}$ & $\mathrm{R}$ & $R$ & $\mathrm{R}$ & $\mathrm{R}$ & $R$ & $\mathrm{R}$ & $\mathrm{R}$ & $\mathrm{R}$ & $R$ & $\mathrm{~S}$ & $\mathrm{R}$ & $S$ \\
\hline $66 \mathrm{I}(\mathrm{CL})$ & $\begin{array}{c}\text { K. } \\
\text { pneumoniae }\end{array}$ & $\begin{array}{c}\text { OXA-48, } \\
\text { CTX-M-15, } \\
\text { SHV- } 1 / 28\end{array}$ & $\mathrm{~S}$ & $\mathrm{R}$ & $\mathrm{R}$ & $\mathrm{R}$ & $\mathrm{R}$ & $\mathrm{R}$ & $\mathrm{R}$ & S & $\mathrm{R}$ & $\mathrm{R}$ & $\mathrm{R}$ & $\mathrm{S}$ & $\mathrm{R}$ & $\mathrm{S}$ & $\mathrm{R}$ & $\mathrm{S}$ & $\mathrm{R}$ & $\mathrm{S}$ & $\mathrm{R}$ & $R$ \\
\hline $601(\mathrm{CL})$ & M. morganii & $\begin{array}{c}\text { OXA-48, } \\
\text { CTX-M-15, } \\
\text { SHV-12 }\end{array}$ & S & $\mathrm{R}$ & $\mathrm{R}$ & $R$ & $\mathrm{R}$ & $R$ & $\mathrm{R}$ & $\mathrm{R}$ & S & S & $\mathrm{R}$ & $R$ & $R$ & S & $R$ & $\mathrm{R}$ & $R$ & $R$ & $\mathrm{R}$ & $\mathrm{R}$ \\
\hline $51 \mathrm{I}(\mathrm{F})$ & A. baumannii & $\begin{array}{l}\text { OXA-23, } \\
\text { SHV-12 }\end{array}$ & NT & $\mathrm{R}$ & NT & NT & NT & NT & $R$ & S & $R$ & $R$ & $R$ & $R$ & $R$ & $\mathrm{R}$ & NT & S & $R$ & NT & S & $R$ \\
\hline 45 I (CL) & A. baumannii & OXA-23 & $\mathrm{S}$ & $R$ & NT & NT & NT & NT & $\mathrm{S}$ & $\mathrm{S}$ & $\mathrm{R}$ & $\mathrm{S}$ & $\mathrm{S}$ & $R$ & $R$ & NT & NT & $\mathrm{S}$ & $\mathrm{R}$ & NT & $\mathrm{S}$ & $\mathrm{S}$ \\
\hline $41 \mathrm{I}(\mathrm{F})$ & P. aeruginosa & $\begin{array}{l}\text { OXA-10, } \\
\text { VEB-1a, } \\
\text { SHV-12 }\end{array}$ & NT & $R$ & $\mathrm{R}$ & $\mathrm{R}$ & NT & $\mathrm{R}$ & $\mathrm{R}$ & S & $\mathrm{R}$ & $\mathrm{R}$ & $\mathrm{R}$ & $\mathrm{R}$ & $\mathrm{R}$ & $R$ & $R$ & S & $\mathrm{R}$ & NT & $R$ & NT \\
\hline $571(F)$ & E. coli & $\begin{array}{l}\text { VIM-2, } \\
\text { SHV-12 }\end{array}$ & $\mathrm{S}$ & $R$ & $R$ & $R$ & $R$ & $R$ & $R$ & S & $R$ & $R$ & $R$ & $\mathrm{R}$ & $\mathrm{R}$ & $R$ & $R$ & S & $R$ & $\mathrm{R}$ & $R$ & $\mathrm{R}$ \\
\hline
\end{tabular}

AMK: amikacin; ATM: aztreonam; CAZ: ceftazidime; CIP: ciprofloxacin; CL: clinical isolate; CST: colistin; CTX: cefotaxime; DOR: doripenem; DOX: doxycycline; F: faecal isolate; FEP: cefepime; GEN: gentamicin; I: intermediate susceptibility; IPM: imipenem; LVX: levofloxacin; MEM: meropenem; NT: not tested; PMB: polymyxin B; R: resistant; SAM: ampicillin/sulbactam; TGC: tigecycline; TIM: ticarcillin/clavulanic acid; TOB: tobramycin; S: susceptible; SXT: trimethoprim/sulfamethoxazole; TZP: piperacillin/tazobactam.

European Committee on Antimicrobial Susceptibility (EUCAST) breakpoints [4] were used for all antimicrobials except for polymyxin B, for which Clinical and Laboratory Standard Institute (CLSI) interpretative criteria [10,11] were used.

carbapenemases were not identified in any of the hospitals investigated.

Previously unreported species/genes combinations were also identified in Hospital 3. For instance, $A$. baumannii isolates co-harboured bla $a_{\mathrm{OXA}-23}$ as well as bla $a_{\mathrm{SHV}-1 / 28}$, while one $E$. coli isolate obtained from faecal screening co-harboured bla $a_{\mathrm{VIM}-2}$ and $b l a_{\mathrm{SHV}-12}$. All except two isolates from Hospital 3 that carried a gene associated with carbapenem resistance also co-produced CTX-M-15 and/or SHV-12 beta-lactamases.
Overall, of the 50 CP-GNB identified in this study, 27 were from clinical specimens, 23 were obtained through faecal screening and three from environmental samples. Two patients with $A$. baumannii OXA-23producing infections (pneumonia, bacteraemia) had faecal colonisation with the same CP-GNB. Another patient was found to be colonised with multiple CP-GNBs, where OXA-23-producing $A$. baumannii and OXA-48-producing $K$. pneumoniae were isolated from a wound infection while OXA-10-producing $P$. aeruginosa was detected through faecal screening. 
Susceptibility testing showed variable levels of resistance to carbapenems, spanning from NDM-1-producing S. marcescens and OXA-48-producing $K$. pneumoniae exhibiting susceptibility to doripenem, imipenem and meropenem through to $P$. aeruginosa co-producing OXA-10, VEB-1a and SHV-12 beta-lactamases, which manifested resistance to all antimicrobials tested except for polymyxin B and colistin (Table). Taken together, these results highlight the difficulties faced by laboratories that only rely on phenotypic methods for detection of carbapenemases.

Due to the multidrug-resistant profiles of many clinical isolates, a suitable recipient for conjugation experiments was identified for only five isolates (one NDM1-producing $S$. marcescens and two NDM-1-producing K. pneumoniae from Hospital 1 and two OXA-48producing $K$. pneumoniae from Hospital 3); conjugative plasmids were successfully transferred in all five isolates. PBRT showed that bla $a_{\mathrm{NDM}-1}$ was associated with IncFlls plasmids in both $K$. pneumoniae and S. marcescens from Hospital 1. However, PBRT performed on clinical Serratia NDM-1-positive isolates also identified IncM plasmids in five isolates and $\mathrm{InCL}$ in three isolates, while no typable plasmids could be found in six clinical isolates carrying bla $a_{\mathrm{NDM}-1}$. IncL plasmids were found to be associated with bla $a_{0 \times A-48}$ in K. pneumoniae clinical isolates and transconjugants from resistancetransfer studies. In addition, IncL was the only plasmid found in all $K$. pneumoniae clinical isolates that carried bla $a_{\text {OXA-48 }}$ from both hospitals (Hospitals 1 and 3). PCR also showed that no ESBL-resistance determinants had co-transferred with the carbapenem-resistance genes.

\section{Discussion}

This pilot study demonstrated a high prevalence of CP-GNB in two of three Romanian hospitals over a six-month period. Furthermore, a variety of carbapenemase-producing species and genes associated with carbapenem resistance were also identified in this study. The distribution of the CP-GNB varied between the two hospitals in which these bacteria were detected: NDM-1-producing S. marcescens and $K$. pneumoniae were prevalent in Hospital 1 , in the north-central part of the country, but were not identified in Hospital 3, in the north-east. No history of travel abroad could be identified in the patients and the origin of the NDM-1 isolates needs further investigation to understand the epidemiology of NDM-1-producing Enterobacteriaceae in eastern Europe [16].

In our study, bla $a_{\mathrm{NDM}-1}$ was identified in 17 isolates of $S$. marcescens across clinical, environmental and faecal isolates, all of which were obtained from the same neonatal unit, suggesting the likelihood of an outbreak, which will be further investigated. This is the largest number of NDM-1-producing $S$. marcescens detected in one hospital in Europe, with only one previous report of a clinical isolate in Germany [17], highlighting the spread of bla $a_{\mathrm{NDM}-1}$ into another Enterobacteriaceae species. Although an opportunistic pathogen, $S$. marcescens has emerged in the past decades as an important source of hospital acquired-infections and outbreaks in both adult and paediatric intensive-care patients $[18,19]$. Hospital 1, where the NDM-1-producing $S$. marcescens and $K$. pneumoniae were isolated, had previous Serratia spp. outbreaks during 2010-12 in the same neonatal unit with Serratia spp. isolates that did not produce carbapenemase (data not shown). Our conjugation studies identified $b a_{\text {NDM-1 }}$ to be associated with conjugative IncFlls plasmids in both $K$. pneumoniae and S. marcescens, which could suggest interspecies transfer of $b a_{\mathrm{NDM}-1}$ via mobile genetic elements. Whole genome sequencing will be carried out of the NDM-1-negative and NDM-1-producing Serratia and Klebsiella isolates obtained in this study, as well isolates from previous outbreaks in this neonatal unit, to understand the molecular epidemiology of NDM-1producing S. marcescens in this hospital and in surrounding areas in eastern Europe.

In contrast, NDM-1 was not found in Hospital 3, whereas OXA-23- and OXA-24-producing $A$. baumannii, OXA-48producing K.pneumoniae and $M$. morganii, as well as OXA-10-producing $P$. aeruginosa, were the most prevalent in this hospital. Interestingly, the bla $a_{0 \times A-10}$-carrying $P$. aeruginosa isolates exhibited resistance to all tested carbapenems, which could imply that OXA-10 betalactamases in Pseudomonas spp. may have catalytic activity against carbapenems, as has been demonstrated for Acinetobacter spp. [9]. Further research is necessary, however, to establish the ability of OXA-10 beta-lactamases to compromise therapeutic effectiveness of carbapenems in Pseudomonas spp. OXA-48producing $K$. pneumoniae was the most common CPE in Hospital 3 and conjugation studies identified bla ${ }_{\text {oxA-48 }}$ to be associated with IncL plasmids. Previous studies have shown that plasmids carrying bla $a_{\mathrm{OXA}-48}$ share similar features, in that they have an IncL/M backbone and are very similar in size (about 60-70 kb) suggesting their wide dissemination in different countries through an epidemic plasmid [20]. A recent study specifically assigned bla $a_{\text {OXA-48 }}$-carrying plasmids to the InCL group [15]; in our study, we demonstrated the association of bla $a_{0 \times 48}$ with IncL plasmids in all carbapenemase-producing $K$. pneumoniae isolates from two hospitals in different geographical regions in Romania.

Previously unreported species/genes combinations, or bacteria co-harbouring more than one carbapenemse-encoding gene, were also identified in Hospital 3. Detection of a patient colonised with $E$. coli-producing VIM-2 is the first known report of $E$. coli harbouring bla $a_{\mathrm{VIM}-2}$ in a hospital patient in Romania, following previous detection of this genotype in patients from Germany between September 2009 and May 2013 [21]. Our study also detected M. morganii-producing OXA48, a CP-GNB that has not been previously reported in Europe, but has been described in clinical isolates from Lebanon and Kuwait $[22,23]$. 
A. baumannii is known to be an agent of nosocomial infections associated with ICUs [24]. It is widely recognised that this bacterium is very well adapted to withstand dry environmental conditions and disinfectants, being able to survive for a long time (several months) on hospital surfaces [24]. Previous molecular analyses of $A$. baumannii isolates from Hospital 3's cardiac ICU obtained in 2011-12, identified widespread infections with OXA-23-producing $A$. baumannii belonging to European clones I and II (data not shown). Such findings should trigger appropriate infection control measures, however, previous studies have shown that very often routine infection control measures are insufficient to control $A$. baumannii and enhanced measures, such as using closed tracheal suction systems, patient isolation or sometimes ward closure, are necessary to control this resilient opportunistic pathogen [24].

There are only a few previous reports on the isolation and/or characterisation of CP-GNB from Romanian hospitals, which described NDM-1-producing E. cloacae, OXA-48-producing $K$. pneumoniae, OXA-23-producing A. baumannii and VIM-2-producing $P$. aeruginosa clinical isolates [25-29]. In one study, Deshpande et al. analysed clinical isolates from three Romanian hospitals in 2011 and found 3\% of isolates from two hospitals with reduced susceptibility to carbapenems, two of which were NDM-1-producing $E$. cloacae, the other being OXA48-producing $K$. pneumoniae [25]. Only a few years later, our study demonstrates a high prevalence of carbapenem non-susceptible Gram-negative isolates, ranging from $5 \%$ to $20 \%$, as well as a great diversity of bacterial species and genes associated with carbapenem resistance. Detection of CP-GNB through the implementation of a patient screening programme demonstrated the presence of these bacteria in clinical and faecal samples of newly admitted hospital patients, providing a snapshot of what is likely to be an endemic spread of CP-GNB in this country. Larger, nationwide screening studies are necessary to determine the true prevalence of CP-GNB in Romanian hospitals.

Our study has some limitations. Firstly, the criteria used to select newly admitted patients for screening (previous hospitalisation, previous infection with multidrug-resistant bacteria or previous antimicrobial treatment) were applied in Hospital 3 but could not be applied in Hospitals 1 and 2, where all patients newly admitted to selected wards were screened. Therefore, risk factors for CP-GNB colonisation of newly admitted patients could not be determined. Secondly, clinical data were not available from Hospitals 1 and 2, therefore risk factors for CP-GNB infection could also not be determined.

Despite these limitations, data from Hospital 3, where screening criteria were used for newly admitted patients, showed that two patients selected for faecal screening had multiple hospitalisations in the previous year, one whom was found to have concurrent OXA-23producing $A$. baumannii faecal and tracheal exudate colonisation. Also, hospital records showed that six patients from whom surveillance cultures yielded faecal carbapenem non-susceptible bacteria had been previously admitted to the same hospital during the past year, during which time they had been treated with imipenem. Of these, one patient had a wound infection with an OXA-48-producing $K$. pneumoniae and also faecal carriage of carbapenem non-susceptible $P$. aeruginosa. Another patient had concurrent faecal and tracheal exudate colonisation, as well as blood cultures containing OXA-23-producing $A$. baumannii. Finally, faecal carriage of VIM-2-producing $E$. coli was found in another patient who had been previously admitted to Hospital 3. These findings highlight the importance of active surveillance of asymptomatic carriers and identification of patients who may have a higher risk of CP-GNB carriage, such as those with previous multiple hospital admissions.

The high prevalence and variety of CP-GNB identified in two of the three hospitals investigated in this study are of concern and suggest the need for a review of the local surveillance and infection control measures. Romania is one of the few European countries that does not currently have a national surveillance system or specific guidelines for the management of CP-GNB infections, nor are hospitals obliged to report such infections to the health authorities [30]. As a consequence, the importance of these infections may be underestimated by hospital authorities. In the hospitals investigated, the ICUs had no facilities for patient isolation and at best, mobile curtains are used to separate beds. Although financial constraints may not allow infrastructure changes that would ensure strict patient isolation, more could be done to educate staff and implement feasible measures to prevent the introduction and spread of CP-GNB. Therefore, reinforcement of measures such as staff education on hand hygiene and contact precautions combined with active faecal culture surveillance are likely to have a direct impact on preventing the spread of CP-GNB and thus reducing the risk of such infections. Introduction of mandatory screening and reporting of CP-GNB by hospitals, as part of a coordinated surveillance programme, should provide an effective framework for the early detection and control of CP-GNB.

In conclusion, our findings emphasise the need for early detection of patients with CP-GNB colonisation or infection, which should lead to the implementation of appropriate infection control measures to prevent the silent spread of these isolates in hospitals. Improvement of surveillance of carbapenemase-producing bacteria in Romania - including introducing guidelines for laboratory detection of intestinal asymptomatic carriers and for infection control and outbreak management - will not only benefit the local patients but will also have an impact at European level, as it is recognised that the spread of carabapenemase-producing organisms is often linked to travel of patients between various countries [16]. 


\section{Acknowledgements}

This work was supported by a grant from the Microbiology Society (International Development Fund).

\section{Conflict of interest}

None declared.

\section{Authors' contributions}

DT coordinated the study, analysed data, wrote the manuscript; CVP and MD assisted in designing the study, organised implementation of surveillance of carbapenemase-producing bacteria in one of the hospitals collected clinical data, read and revised the manuscript; IEM assisted in designing the study, performed molecular testing, analysed data, read and revised manuscript; $A D M, A M$ and FT assisted in designing the study, organised implementation of surveillance of carbapenemase-producing bacteria in two of the hospitals, collected clinical data, read and revised the manuscript.

\section{References}

1. Bakour S, Olaitan AO, Ammari H, Touati A, Saoudi S, Saoudi $\mathrm{K}$, et al. Emergence of Colistin- and Carbapenem-Resistant Acinetobacter baumannii ST2 Clinical Isolate in Algeria: First Case Report. Microb Drug Resist. 2015;21(3):279-85. DOI: 10.1089/mdr.2014.0214 PMID: 25588125

2. Glasner C, Albiger B, Buist G, Tambić Andrasević A, Canton $R$, Carmeli Y, et al. , European Survey on CarbapenemaseProducing Enterobacteriaceae (EuSCAPE) Working Group. Carbapenemase-producing Enterobacteriaceae in Europe: a survey among national experts from 39 countries, February 2013. Euro Surveill. 2013;18(28):20525. DOI: 10.2807/15607917.ES2013.18.28.20525 PMID: 23870096

3. European Centre for Disease Prevention and Control (ECDC). Antimicrobial resistance surveillance in Europe 2012. Annual report of the European Antimicrobial Resistance Surveillance Network (EARS-Net). Stockholm: ECDC; 2013. Available from: http://ecdc.europa.eu/en/publications/Publications/ antimicrobial-resistance-surveillance-europe-2012.pdf

4. European Committee on Antimicrobial Susceptibility Testing (EUCAST). Guidelines for detection of resistance mechanisms and specific resistances of clinical and/or epidemiological importance. Version 1.0. EUCAST. [Accessed 19 Jan 2016]. Available from: http://www.eucast.org/fileadmin/src/media/ PDFs/EUCAST files/Resistance mechanisms/EUCAST detection_of_resistance_mechanisms_v1.0_20131211.pdf

5. Dallenne C, Da Costa A, Decré D, Favier C, Arlet G. Development of a set of multiplex PCR assays for the detection of genes encoding important beta-lactamases in Enterobacteriaceae.) Antimicrob Chemother. 2010;65(3):490-5. DOI: 10.1093/jac/ dkp498 PMID: 20071363

6. Corvec S, Caroff N, Espaze E, Giraudeau C, Drugeon H, Reynaud A. AmpC cephalosporinase hyperproduction in Acinetobacter baumannii clinical strains.J Antimicrob Chemother. 2003;52(4):629-35. DOI: $10.1093 / \mathrm{jac} / \mathrm{dkg} 407$ PMID: 12951337

7. Nordmann P, Poirel L, Carrër A, Toleman MA, Walsh TR. How to detect NDM-1 producers.J Clin Microbiol. 2011;49(2):718-21. DOI: 10.1128/JCM.01773-10 PMID: 21123531

8. Woodford N, Ellington MJ, Coelho JM, Turton JF, Ward ME, Brown S, et al. Multiplex PCR for genes encoding prevalent OXA carbapenemases in Acinetobacter spp. Int j Antimicrob Agents. 2006;27(4):351-3. DOI: 10.1016/j. ijantimicag.2006.01.004 PMID: 16564159

9. Antunes NT, Lamoureaux TL, Toth M, Stewart NK, Frase $\mathrm{H}$, Vakulenko SB. Class D $\beta$-lactamases: are they all carbapenemases?Antimicrob Agents Chemother. 2014;58(4):2119-25. DOI: 10.1128/AAC.02522-13 PMID: 24468778

10. European Committee on Antimicrobial Susceptibility Testing (EUCAST). Breakpoint tables for interpretation of MICs and zone diameters. Version 5.0, 2015. EUCAST; 2015. Available from: http://www.eucast.org/fileadmin/src/media/PDFs/ EUCAST_files/Breakpoint_tables/v_5.0_Breakpoint_Table_01. pdf
11. Clinical and Laboratory Standard Institute (CLSI). Performance standards for antimicrobial susceptibility testing. Twenty-first informational supplement. M100-S21. Vol. 31 No. 1. CLSI;2011.

12. Oliver A, Weigel LM, Rasheed JK, McGowan JE, Raney P, Tenover FC. Mechanisms of decreased susceptibility to cefpodoxime in Escherichia coli.Antimicrob Agents Chemother. 2002;46(12):3829-36. DOI: 10.1128/AAC.46.12.3829-3836.2002 PMID: 12435684

13. McDaniels AE, Rice EW, Reyes AL, Johnson $\mathrm{CH}$, Haugland RA, Stelma GN. Confirmational identification of Escherichia coli, a comparison of genotypic and phenotypic assays for glutamate decarboxylase and beta-D-glucuronidase.Appl Environ Microbiol. 1998;64(10):4113.PMID: 16349569

14. Carattoli A, Bertini A, Villa L, Falbo V, Hopkins KL, Threlfall EJ. Identification of plasmids by PCR-based replicon typing. Microbiol Methods. 2005;63(3):219-28. DOI: $10.1016 / \mathrm{j}$. mimet.2005.03.018 PMID: 15935499

15. Carattoli A, Seiffert SN, Schwendener S, Perreten V, Endimiani A. Differentiation of InCL and IncM Plasmids Associated with the Spread of Clinically Relevant Antimicrobial Resistance.PLoS One. 2015;10(5):e0123063. DOI: 10.1371/journal.pone.0123063 PMID: 25933288

16. Livermore DM, Walsh TR, Toleman M, Woodford N. Balkan NDM-1: escape or transplant?Lancet Infect Dis. 2011;11(3):164. DOI: $10.1016 /$ S1473-3099(11)70048-2 PMID: 21371654

17. Gruber TM, Göttig S, Mark L, Christ S, Kempf VAJ, Wichelhaus TA, et al. Pathogenicity of pan-drug-resistant Serratia marcescens harbouring blaNDM-1. J Antimicrob Chemother. 2015;70(4):1026-30.PMID: 25468904

18. Voelz A, Müller A, Gillen J, Le C, Dresbach T, Engelhart S, et al. Outbreaks of Serratia marcescens in neonatal and pediatric intensive care units: clinical aspects, risk factors and management. Int J Hyg Environ Health. 2010;213(2):79-87. DOI: 10.1016/j.ijheh.2009.09.003 PMID: 19783209

19. Yoon HJ, Choi JY, Park YS, Kim CO, Kim JM, Yong DE, et al. Outbreaks of Serratia marcescens bacteriuria in a neurosurgical intensive care unit of a tertiary care teaching hospital: a clinical, epidemiologic, and laboratory perspective. Am J Infect Control. 2005;33(10):595-601. DOI: 10.1016/j. ajic.2005.01.010 PMID: 16330308

20. Poirel L, Bonnin RA, Nordmann P. Genetic features of the widespread plasmid coding for the carbapenemase OXA 48. Antimicrob Agents Chemother. 2012;56(1):559-62. DOI: 10.1128/AAC.05289-11 PMID: 22083465

21. Kaase M, Pfennigwerth N, Lange F, Anders A, Gatermann SG. Molecular epidemiology of VIM-1 producing Escherichia coli from Germany referred to the National Reference Laboratory. Int J Med Microbiol. 2015;305(7):784-9. DOI: 10.1016/j. ijmm.2015.08.032 PMID: 26321009

22. Jamal WY, Albert MJ, Khodakhast F, Poirel L, Rotimi VO. Emergence of New Sequence Type OXA-48 CarbapenemaseProducing Enterobacteriaceae in Kuwait.Microb Drug Resist. 2015;21(3):329-34. DOI: 10.1089/mdr.2014.0123 PMID: 25551428

23. Hammoudi D, Ayoub Moubareck C, Aires J, Adaime A, Barakat A, Fayad N, et al. Countrywide spread of OXA-48 carbapenemase in Lebanon: surveillance and genetic characterization of carbapenem-non-susceptible Enterobacteriaceae in 10 hospitals over a one-year period. Int J Infect Dis. 2014;29:139-44. DOI: 10.1016/j.ijid.2014.07.017 PMID: 25449248

24. Towner KJ. Acinetobacter: an old friend, but a new enemy.J Hosp Infect. 2009;73(4):355-63. DOI: 10.1016/j. jhin.2009.03.032 PMID: 19700220

25. Deshpande LM, Flonta M, Jones RN, Castanheira M. Detection of NDM-1-producing Enterobacteriaceae in Romania: report of the SENTRY Antimicrobial Surveillance Program.J Med Microbiol. 2014;63(Pt 3):483-4. DOI: 10.1099/jmm.0.070334-0 PMID: 24346066

26. Gheorghe I, Novais Â, Grosso F, Rodrigues C, Chifiriuc MC Lazar V, et al. Snapshot on carbapenemase-producing Pseudomonas aeruginosa and Acinetobacter baumannii in Bucharest hospitals reveals unusual clones and novel genetic surroundings for blaOXA-23. J Antimicrob Chemother. 2015;70(4):1016-20.PMID: 25630639

27. Székely E, Damjanova I, Jánvári L, Vas KE, Molnár S, Bilca DV, et al. First description of bla(NDM-1), bla(OXA-48), bla(OXA-181) producing Enterobacteriaceae strains in Romania. Int J Med Microbiol. 2013;303(8):697-700. DOI: 10.1016/j. ijmm.2013.10.001 PMID: 24183483

28. Bonnin RA, Poirel L, Licker M, Nordmann P. Genetic diversity of carbapenem-hydrolysing $\beta$-lactamases in Acinetobacter baumannii from Romanian hospitals.Clin Microbiol Infect. 2011;17(10):1524-8. DOI: 10.1111/j.1469-0691.2011.03622.x PMID: 21883667 
29. Mereuta AI, Badescu AC, Domeanu OS, Iancu LS,

Tuchilus CG. Spread of VIM-2 metallo-beta-lactamase in

Pseudomonas aeruginosa and Acinetobacter baumannii

clinical isolates from lasi, Romania.Rev Rom De Med De

Laborator.2013;21(4):423-30. Available from: https://

www.degruyter.com/dg/viewarticle.fullcontentlink:pdf

eventlink/\$002fj\$002frrlm.2013.21.issue-4\$002frrlm-

2013-0035\$002frrlm-2013-0035.pdf/rrlm-2013-0035.

pdf?t:ac=j\$002frrlm.2013.21.issue-4\$002frrlm-2013-

0035\$002frrlm-2013-0035.xm

30. Albiger B, Glasner C, Struelens MJ, Grundmann H,

Monnet DL, European Survey of Carbapenemase-

Producing Enterobacteriaceae (EuSCAPE) working group.

Carbapenemase-producing Enterobacteriaceae in Europe:

assessment by national experts from 38 countries, May 2015

Euro Surveill. 2015;20(45):30062. DOI: 10.2807/1560-7917.

ES.2015.20.45.30062 PMID: 26675038

\section{License and copyright}

This is an open-access article distributed under the terms of the Creative Commons Attribution (CC BY 4.0) Licence. You may share and adapt the material, but must give appropriate credit to the source, provide a link to the licence, and indicate if changes were made.

This article is copyright of the authors, 2016. 